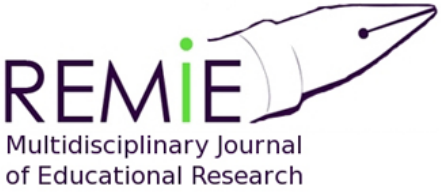

Multidisciplinary Journal of Educational Research

\section{Hipatia Press}

www.hipatiapress.com

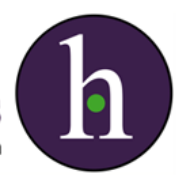

Instructions for authors, subscriptions and further details:

http://remie.hipatiapress.com

\title{
Bullying y Género. Prevención desde la Organización Escolar.
}

Elena Duque ${ }^{1} \&$ Joan Teixido ${ }^{1}$

1) Universidad de Girona, España.

Date of publication: June $15^{\text {th }}, 2016$

Edition period: June 2016-October 2016

To cite this article: Duque, E., \& Teixido, J. (2016). Bullying y Género.

Prevención desde la Organización Escolar. REMIE-Multidisciplinary Journal of Educational Research, 6(2), 176-204.

doi:10.17583/remie.2016.2108

To link this article: http://dx.doi.org/10.17583/remie.2016.2108

\section{PLEASE SCROLL DOWN FOR ARTICLE}

The terms and conditions of use are related to the Open Journal System and to Creative Commons Attribution License (CC-BY). 


\section{Bullying and Gender. Prevention from School Organization}

Elena Duque

Universidad de Girona
Joan Teixido

Universidad de Girona

\section{Abstract}

Homophobia, biphobia, transphobia and gender violence in sexual-affective relationships appear frequently in the bases of bullying. The educational centres are more conscious about this reality and look for actions to prevent and eradicate this kind of violence. At the same time, international research states that bullying prevention needs a re-organization of the educational centre incorporating the work of all the educational community (students, family, teachers, etc.). In this article we gather data on bullying and its connection to gender, part of the Spanish legislation related to this problem is analysed and we conducted an international literature review. The main contribution of this article is to present orientations and actions that prevent and contribute to eradicate gender violence and violence for homophobia, biphobia and transphobia through the school organization and school management. Some of these actions are inclusive education, democratization of the centres, making visible and not trivializing violence, and mainly the community participation and active positioning of all the community against bullying and in favour of the victims.

Keywords: bullying, violencia de género, LGTB, convivencia, organización escolar, comunidad 


\section{Bullying y Género. Prevención desde la Organización Escolar}

Elena Duque

Universidad de Girona
Joan Teixido

Universidad de Girona

\section{Resumen}

La violencia por homofobia, bifobia, transfobia y la violencia de género en relaciones afectivo-sexuales aparecen con frecuencia en la base del acoso escolar. Los centros educativos cada vez son más conscientes de esta realidad y buscan actuaciones que prevengan y erradiquen dicha violencia. Paralelamente las investigaciones internacionales plantean como la prevención del acoso escolar requiere de una reorganización del centro educativo que incorpore el trabajo conjunto de toda la comunidad educativa (alumnado, familiares, profesorado, etc.). En este artículo recogemos datos sobre violencia escolar y su vinculación con el género, analizamos parte de la legislación española relacionada con esta problemática y realizamos una revisión de las investigaciones científicas internacionales al respecto. La contribución principal de este artículo es la presentación de orientaciones y acciones que prevengan y contribuyan a erradicar la violencia de género y la violencia por homofobia, bifobia y transfobia a través de la organización y gestión de los centros educativos. Algunas de estas acciones son la educación inclusiva, la democratización de los centros, la visibilización y no trivialización de la violencia y principalmente la participación y posicionamiento activo de toda la comunidad educativa contra el bullying y a favor de las víctimas.

Palabras clave: bullying, violencia de género, LGTB, convivencia, organización escolar, comunidad 


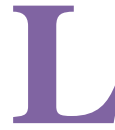

a violencia por cuestiones de género es una grave problemática social. En este marco podemos encontrar tanto violencia de género como homofobia, bifobia y transfobia de las que podemos mostrar algunos datos. La violencia de género es una problemática considerada por la OMS (2013) como un problema de salud global de proporciones epidémicas. En el estudio europeo realizado por la European Union Agency for Fundamental Rights-FRA (2014) se muestra que 1 de cada 3 mujeres ha sufrido violencia física y/o sexual, 1 de cada 10 ha sufrido algún tipo de violencia sexual y 1 de cada 20 ha sido violada. En el ámbito español, la Macroencuesta de Violencia contra las Mujeres (Delegación de Gobierno para la Violencia de Género, 2015) expone que el 24,2\% de mujeres de 16 años o más residentes en España han sido víctimas de violencia física o sexual por parte de su pareja, expareja o terceros. Esta violencia de género también se encuentra muy presente entre jóvenes y menores. Según el Instituto Nacional de Estadística (2015), la tasa de mujeres víctimas de violencia de género a partir de 14 años de edad fue de 1,3 por cada 1.000 . Casi la mitad de las víctimas $(49,3 \%)$ tenían una edad entre 25 y 39 años. El informe sobre Violencia de Género de la Fundación ANAR (Fundación ANAR, 2015) indica que las llamadas de menores alertando sobre violencia de género se han incrementado un $24 \%$ en 2014. Por su parte, el estudio de Díaz-Aguado \& Carvajal (2011) destaca, entre otros datos, que el 4,9\% de las adolescentes ya han sido víctimas de algún tipo de violencia física o psicológica.

En relación a la violencia contra el colectivo $\mathrm{LGTB}^{1}$, esta apenas se recoge en datos estadísticos y los datos oficiales son incompletos (UNFE, n.f). Las Naciones Unidas, a través de su campaña Libres e Iguales ${ }^{2}$ en pro de la igualdad de las personas lesbianas, gays, bisexuales y transgénero (LGBT), afirman que las denuncias por actos de violencia homofóbica y transfóbica se dan en todas las regiones del mundo y que van desde la intimidación psicológica hasta la agresión física, la tortura, el secuestro y el asesinato selectivo, destacando la violencia sexual (UNFE, n.f). Según el Transgender EuroStudy (Whittle, Turner, Combs \& Rhodes, 2008) el 79\% de los transexuales participantes en el estudio habían experimentado algún tipo de acoso en público, desde comentarios transfóbicos hasta abusos físicos o sexuales. Según el informe del Observatori contra l'homofòbia 
(2015) este organismo registró 113 incidencias de LGTBIfobia en Cataluña, identificándose la mayoría de ellas como agresiones $(30,97 \%)$.

Esta violencia presente en toda la sociedad no es ajena a los centros educativos y se halla en muchas situaciones de acoso escolar. Algunas investigaciones centradas en adolescentes han puesto de relieve la relación que existe entre el acoso escolar y el género (Díaz Aguado, 2001, 2004, 2006; Gómez, 2004; Leonardi \& Staley, 2015; McGuire, Anderson, Toomey \& Russell, 2010), pero a pesar de ello una gran parte de los estudios sobre violencia escolar (Banks 1997; Olweus 1993; O’Moore \& Minton 2005; Yoneyama \& Naito, 2003) no contemplan la dimensión de género específicamente.

La preocupación por la violencia escolar no sólo está ocupando el centro de numerosas investigaciones, sino que existen organizaciones civiles, entre las que destacan asociaciones de familiares, que están focalizando en esta problemática y buscando soluciones. La Confederación Española de Asociaciones de Padres (CEAPA) plantea la necesidad de construir escuelas saludables y seguras, incorporando la existencia de políticas educativas que incluyan un plan para mejorar las relaciones interpersonales y evitar situaciones de violencia o acoso (FUNDADEPS, 2012). Por otra parte, existen asociaciones como Chrysallis ${ }^{3}$, asociación de familias de menores transexuales, que en su página web disponen de recursos educativos y materiales que están siendo de utilidad para los centros educativos y las familias que acuden a ella.

Ante esta problemática social, el objetivo de este artículo es presentar acciones preventivas y que contribuyan a la erradicación de la violencia escolar, especialmente en relación al género, desde el ámbito de la organización escolar. Para ello, inicialmente recogemos datos sobre violencia escolar y su vinculación con el género (violencia de género, homofobia, bifobia y transfobia). A continuación realizamos un breve análisis de la legislación educativa y sobre género en relación a la prevención y acción frente a la violencia escolar; y seguidamente llevamos a cabo una revisión de la literatura científica al respecto. Finalmente, en base al análisis previo presentamos algunas orientaciones y acciones educativas para llevar a cabo desde el ámbito de la organización escolar. 


\section{Acoso Escolar y Género en las Aulas}

Internacionalmente, la violencia de género es una preocupante realidad (Srabstein, 2015). Según UNICEF (2014), más de 1 cada 3 estudiantes entre 13 y 15 años en el mundo están sufriendo bullying de forma regular. Amnistía Internacional (2008) hace referencia explícita a la seguridad en las escuelas para las niñas, ya que son las que más violencia reciben en estos contextos. En España, según el Informe Cisneros X (Piñuel \& Oñate, 2007) la violencia en los centros educativos de primaria y secundaria es de un $23,30 \%$ sobre una muestra de 24.490 niños y niñas de 14 comunidades autónomas. En este marco, la creación de centros educativos seguros es un objetivo internacional y europeo. La seguridad del alumnado en los centros educativos, además, está asociada con una mejora del aprendizaje y el rendimiento académico (Devine \& Cohen, 2007; Thapa, Cohen, Guffey \& Higgins-D'Alessandro, 2013), mientras que ser víctima de acoso escolar tiene repercusiones directas no solo en un menor rendimiento académico sino en la generación de una baja autoestima, depresión y ansiedad, entre otras problemáticas (Mayes \& Cohen, 2003; Cava, Buelga, Musitu y Murgui, 2010; Osvaldsson, 2011) En este marco, los centros educativos cada vez están más interesados en incorporar acciones que prevengan y combatan el bullying. El Ministerio de Educación, Cultura y Deporte (MECD), al igual que otros organismos educativos oficiales, recoge en su página web medidas existentes para trabajar esta temática ${ }^{4}$.

Respecto a la cuestión de género en el acoso escolar, diversas investigaciones internacionales nos muestran que la violencia contra las mujeres se da en los diferentes contextos educativos y ponen de manifiesto que actualmente chicos y chicas adolescentes están sufriendo agresiones sexuales por parte de sus iguales en contextos escolares y en sus primeras relaciones afectivas y sexuales (Fineran \& Bennett, 1999; Lavoie, Robitaille \& Hébert, 2000; Silverman, Raj, Mucci \& Hathaway, 2001). Esta violencia, y la existencia de contextos académicos poco favorables para combatirla, tienen repercusiones sobre las mujeres afectadas, a nivel personal o en su rendimiento académico (Wagner \& Magnusson, 2005). En relación al acoso escolar vinculado al colectivo LGTB, McGuire et al. (2010) consideran que este es uno de los grupos con más riesgo de sufrir 
bullying, igual que las minorías culturales, la población inmigrante o las personas con discapacidad. Leonardi y Staley (2015) destacan que las escuelas tienden a ser espacios inseguros e insoportables para este colectivo. Recurriendo a estudios realizados por fundaciones y asociaciones hallamos que en el estudio de la Federación Estatal de Lesbianas, Gays, Transexuales y Bisexuales (2012) sobre acoso escolar homofóbico y el riesgo de suicidio y jóvenes LGTB se muestra que los 653 participantes habían sufrido situaciones de acoso escolar. Entre las formas de violencia sufridas, destacan los insultos (71\%), los rumores (69\%) o el aislamiento (37\%). En relación a la violencia física, a un 36\% le habían tirado cosas o había recibido golpes o empujones, un $23 \%$ de jóvenes declara haber sufrido amenazas, un $6 \%$ había padecido acoso o agresiones sexuales y un $5 \%$ había recibido palizas. Cabe destacar un $14 \%$ que había recibido algún tipo de violencia a través de internet o móvil.

El acoso escolar vinculado a casos de discriminación de género y/o violencia de género se hace también muy evidente a través de los medios de comunicación. En Canadá, por ejemplo, salió a la luz el caso de Amanda Todd (Monge, 2012), adolescente de 15 años que se suicidó en 2012 después de recibir acoso online y escolar, incluso cambiando de centro educativo. Un acoso directamente relacionado con las relaciones afectivosexuales que la víctima había mantenido. Otro ejemplo es el caso de Maore, estudiante de $2^{\circ}$ de Educación Secundaria Obligatoria, asesinada en Barcelona por dos compañeros de clase, con uno de los cuales había mantenido una relación esporádica (Fernández, 2008). En el informe del Observatori de l'Homoföbia (2015) se analizan diferentes casos de violencia, entre los que aparece el suicidio de Alan, un menor transexual que ha sido protagonista de numerosas noticias (Álvarez, 2015). Alan fue acosado en los diferentes centros educativos donde estudió y donde se manifestó primero como lesbiana y después como transexual, siendo el primer menor al que se le fue permitido cambiar su DNI en Cataluña (Piulachs, 2015), y acabó suicidándose en diciembre de 2015. El Observatori de l'Homofòbia (2015) resalta como en los casos de bullying por transfobia es el menor el que tiene que cambiar de centro, siendo la víctima la que se ve obligada a huir de esta situación. Así mismo, se destaca 
que por este motivo es necesario establecer mecanismos de protección de personas LGTBI en los centros educativos.

\section{Metodología}

Este artículo tiene como objetivo presentar orientaciones y acciones que prevengan y contribuyan a erradicar la violencia de género y la violencia por homofobia, bifobia y transfobia a través de la organización y gestión de los centros educativos. Para la consecución de este objetivo hemos realizado un análisis documental en base dos ámbitos principalmente: la legislación española y la literatura científica internacional.

En relación a la legislación, se han escogido tres leyes españolas seleccionadas por estar directamente relacionadas con la temática estudiada en el artículo. En el análisis de estas leyes hemos resaltado los aspectos relativos a la prevención y acción frente al acoso escolar y, en concreto, su vinculación con el género, desde el ámbito de la organización escolar.

Las leyes escogidas han sido: a) la Ley Orgánica 8/2013, de 9 de diciembre, para la mejora de la calidad educativa (LOMCE) ${ }^{5}$, la ley vigente y más reciente sobre el sistema educativo en España; b) la Ley Orgánica 1/2004, de 28 de diciembre, de Medidas de Protección Integral contra la Violencia de Género ${ }^{6}$, ley pionera en Europa y vigente en España; y c) la Ley 11/2014, de 10 de octubre, para garantizar los derechos de lesbianas, gays, bisexuales, transgéneros e intersexuales y para erradicar la homofobia, la bifobia y la transfobia ${ }^{7}$, ley en el ámbito de Cataluña, escogida al no existir una de iguales características en el ámbito estatal.

Respecto a la literatura científica internacional, hemos realizado una revisión bibliográfica a través de revistas científicas indexadas en diferentes bases de datos, destacando entre ellas Journal Citation Report (JCR) y SCOPUS. La búsqueda realizada se ha centrado en aquellas acciones educativas, preventivas y de actuación frente al bullying especialmente relacionados con violencia de género y con colectivo LGTB. 


\section{Acoso Escolar y Género desde la Legislación Española}

La prevención de conflictos, las acciones ante el bullying, y la promoción de una convivencia desde un enfoque en el que participe toda la comunidad educativa están presentes en la legislación española. La Ley Orgánica 8/2013, de 9 de diciembre, para la mejora de la calidad educativa. (LOMCE), en el artículo 1 sobre los principios educativos, explicita los conflictos y en concreto el acoso escolar como uno de los elementos clave a prevenir, además de hacer referencia explícita a la prevención de la violencia de género:

(...) k) La educación para la prevención de conflictos y la resolución pacífica de los mismos, así como para la no violencia en todos los ámbitos de la vida personal, familiar y social, y en especial en el del acoso escolar. 1) El desarrollo, en la escuela, de los valores que fomenten la igualdad efectiva entre hombres y mujeres, así como la prevención de la violencia de género. (p. 97867)

Así mismo, el artículo 124, sobre normas de organización, funcionamiento y convivencia, califica el acoso en general, y en concreto el basado en el género, orientación o identidad sexual, como de falta muy grave:

Aquellas conductas que atenten contra la dignidad personal de otros miembros de la comunidad educativa, que tengan como origen o consecuencia una discriminación o acoso basado en el género, orientación o identidad sexual, (...) tendrán la calificación de falta muy grave y llevarán asociada como medida correctora la expulsión, temporal o definitiva, del centro. (p.97903)

En este mismo artículo se establece la obligatoriedad de elaborar planes de convivencia, y la especial atención que se ha de dar a elementos como la violencia de género:

Los centros elaborarán un plan de convivencia que incorporarán a la programación general anual y que recogerá todas las actividades que se programen con el fin de fomentar un buen clima de convivencia dentro del centro escolar, (...) y la realización de actuaciones para la resolución pacífica de conflictos con especial atención a las actuaciones de prevención de la violencia de género, igualdad y no discriminación. (p. 97903) 
De hecho, según la LOMCE, la prevención de la violencia de género ha de estar tratada tanto en las normas generales de organización y funcionamiento del centro educativo - tal como se establece en el Artículo 124- como en el currículum escolar - tal como se establece en la Disposición adicional cuadragésima primera-. Así mismo, organismos específicos como en el Consejo Escolar - tal como se establece en el artículo 17- tienen el deber de asegurar la prevención de la violencia de género.

En la Ley Orgánica 1/2004, de 28 de diciembre, de Medidas de Protección Integral contra la Violencia de Género se realizan diversas referencias al mundo educativo y escolar. Por un lado, se establece que el sistema educativo debe tratar el tema de la igualdad de género desde la educación infantil; así mismo, se contempla la necesidad de que las Administraciones educativas adopten las medidas necesarias para la formación inicial y permanente en materia de igualdad:

a) La educación en el respeto de los derechos y libertades fundamentales y de la igualdad entre hombres y mujeres y en el ejercicio de la tolerancia y de la libertad dentro de los principios democráticos de convivencia.

b) La educación en la prevención de conflictos y en la resolución pacífica de los mismos, en todos los ámbitos de la vida personal, familiar y social. (p. 7-8)

Los artículos 8 y 9 de esta misma Ley plantean además la importancia de la participación de los Consejos Escolares en la adopción de medidas educativas para fomentar la igualdad real y efectiva entre hombres y mujeres. La inspección educativa también debe velar por el cumplimiento y aplicación de estos principios en el sistema educativo.

Por último, y a falta de una ley estatal, destacamos en el ámbito de Cataluña, la Ley 11/2014, de 10 de octubre, para garantizar los derechos de lesbianas, gays, bisexuales, transgéneros e intersexuales y para erradicar la homofobia, la bifobia y la transfobia, que destaca la necesidad de sensibilización y formación de los profesionales de la educación. El artículo 12 de dicha ley se especializa en el ámbito educativo. En dicho artículo, se plantea la necesidad de eliminar cualquier tipo de discriminación por razón de orientación sexual, identidad de género o expresión de género; así como de velar porque la diversidad sexual y afectiva, la identidad de género y los diferentes modelos de familia sean respetados en los distintos ámbitos 
educativos, evitando así cualquier tipo de discriminación en los contenidos de los materiales escolares. También se hace referencia a la educación no formal y la educación en el tiempo libre. Respecto a la convivencia escolar y la acción de toda la comunidad educativa destacamos lo siguiente:

6. Debe velarse por la concienciación y la prevención de la violencia por razón de orientación sexual, identidad de género o expresión de género y ofrecer mecanismos a los centros para que detecten situaciones de discriminación o exclusión de cualquier persona por las dichas razones. En este sentido, debe promoverse el desarrollo efectivo de planes de convivencia con un especial énfasis en las medidas de prevención y de actuación contra el acoso de que pueden ser objeto las personas LGBTI en el medio escolar.

7. (...) debe velar porque las escuelas, los institutos y los otros centros educativos constituyan un entorno amable para la diversidad sexual y afectiva en el que alumnos y profesores puedan vivir de una manera natural su orientación sexual, identidad de género o expresión de género (...) (p. 94736)

\section{Organización Escolar y Participación de toda la Comunidad}

Aunque existen acciones puntuales que pueden mejorar la convivencia, la mayoría de investigaciones plantean que el acoso escolar tiene que ser abordado de forma global y con la intervención de toda la comunidad. Autores como Torrego y Martínez (2014) resaltan que la convivencia mejora cuando hay un plan de intervención global. Por su parte, Gairín, Armengol y Silva (2013) proponen que la convivencia sea un aspecto a considerar en la organización y funcionamiento del centro educativo. Así mismo, el modelo dialógico de resolución de conflictos, una actuación educativa de éxito avalada por la comunidad científica internacional (Flecha, 2015), parte de la implicación de toda la comunidad y de la reorganización de los recursos y la organización del centro educativo. En este sentido, se hace necesario que el centro se organice contando con la participación decisiva de toda la comunidad educativa, y que a su vez se persiga este objetivo común de mejora de la convivencia. De hecho, según Teixido \& Castillo (2010), la dimensión organizativa es una de las dimensiones a trabajar para mejorar la convivencia de los centros ya que el 
modelo organizativo de un centro no es neutro, sino que transmite valores y actitudes que pueden favorecer o dificultar la consolidación de un clima de convivencia. Según estos mismos autores es necesario configurar los diversos aspectos que delimitan el funcionamiento de un centro teniendo en cuenta si favorecen o dificultan los procesos de resolución de conflictos, la participación de las familias y el alumnado en la gestión del centro y la contribución a crear un sentimiento de comunidad, entre otros.

La importancia de la implicación de toda la comunidad educativa para mejorar la convivencia es clave. Uno de los ejes principales en este sentido es la participación de las familias, que ha sido identificada por diferentes investigaciones científicas como un aspecto esencial para la mejora de la educación (Edwards \& Warin, 1999; Epstein, 2001; Sanders \& Sheldon, 2009; Sénechal \& LeFevre, 2002). Algunos autores destacan que el diálogo que se establece entre el centro educativo y las familias tiene que llegar a todas las familias, especialmente a aquellas más desfavorecidas (Espelage, et al, 2013; Flecha, 2015). En el ámbito de género, se destaca que el hecho de dar protagonismo a todas las mujeres, especialmente a aquellas no académicas, es decir, que carecen de estudios superiores (Oliver, Soler \& Flecha, 2009) contribuye a la detección y prevención de situaciones de violencia de género. En este sentido, también es clave la participación de los padres. Según la investigación de Gómez, Munté y Sordé (2014), la participación de padres y/o familiares hombres gitanos y de origen marroquí en la escuela contribuye a superar las imágenes sexistas y racistas estereotipadas hacia estos colectivos. Además, según Redondo-Sama, Rodríguez-Pulido, Larena y De Botton (2014), la interacción de los y las estudiantes con personas de diversas culturas contribuye a romper con las barreras simbólicas que existen entre la institución educativa y determinadas familias, facilitándose así la participación.

Dentro del centro educativo, además de la inclusión de familiares y otros miembros de la comunidad educativa, es necesaria la cohesión del profesorado y la implicación del equipo directivo y, en concreto, del director o directora del centro. Las investigaciones internacionales muestran la vinculación entre el liderazgo educativo y la mejora de la educación (Leithwood, Day, Sammons, Harris \& Hopkins, 2006; Witziers, Bosker \& Krüger, 2003), tanto en los que respecta a los resultados académicos como 
en la convivencia (Robinson, Lloyd \& Rowe, 2008). Así mismo, esta mejora de la educación puede ir más allá del centro educativo e incidir en la mejora de la comunidad educativa en general (Houston, Blankstein \& Cole, 2010; Sanders \& Harvey, 2002). Algunos autores (Teixido \& Capell, 2006; Teixido \& Castillo, 2010) subrayan la importancia de la implicación de los directores y directoras en la creación de un clima de convivencia más y concretamente ante la violencia escolar, democratizando el centro educativo y promoviendo un equipo directivo sólido y cohesionado. Así mismo, ante la violencia escolar se debe intervenir no solo con un sector del alumnado en concreto, sino en el ámbito de la clase y en el de toda la comunidad educativa. En este sentido, Torrego y Martínez (2014) resaltan la necesidad de un liderazgo institucional, pedagógico y emocional que establezca las condiciones organizativas necesarias para llevar a cabo un plan de convivencia. Así mismo, destacan la necesidad de crear un clima de confianza y seguridad desde un estilo democrático de escuela, que cuente con la participación de los distintos miembros de la comunidad educativa. La importancia de generar un liderazgo educativo unido a la participación de la comunidad es planteado desde otros autores desde el enfoque del liderazgo dialógico (Padrós \& Flecha, 2014; Redondo-Sama, 2015). Dicho liderazgo se crea y se consolida mediante la participación decisiva y educativa de todos los miembros de la comunidad educativa, es decir, la participación en la toma de decisiones del centro educativo y en el ámbito educativo.

La participación de la comunidad educativa en la mejora de la convivencia y en la prevención de la violencia escolar pasa también por la implicación del alumnado en las acciones educativas del centro. Por un lado, cabe destacar que la relación positiva entre profesorado-alumnado genera dinámicas de apoyo que inciden en la seguridad del alumnado y la buena convivencia (Gregory \& Cornell, 2009; Jia et al. 2009; Wang, Selman, Dishion, \& Stormshak, 2010). Por otro lado, los estudios indican que las relaciones entre las y los iguales son la clave para potenciar un clima de convivencia positivo (Guo \& Higgins-D’Alessandro, 2011). Cuando nos encontramos en situaciones de violencia entre iguales, la literatura científica plantea como es entre el grupo de iguales como más se puede incidir en prevenir y erradicar dicha violencia. La solidaridad y la 
amistad dentro del grupo desempeñan un papel clave (Banyard, et al, 2005; Fisher, Daigle \& Cullen, 2010; Gross, Winslett, Roberts \& Gohm, 2006). Por ejemplo, Boulton (1999) destaca en los resultados de su estudio que las y los pre-adolescentes reciben menos ataques y acosos cuando tienen más relaciones de amistad en su grupo-clase. Algunos estudios plantean la importancia de la bystander intervention, es decir, la protección que dan los propios compañeros y compañeras del grupo-clase que son testigos del bullying, y actúan apoyando y defendiendo a las víctimas, creando espacios solidarios. De esta manera, las compañeras y compañeros como testigos de una situación de violencia escolar, la rechazan y la denuncian, en vez de quedarse en silencio (Thapa et al., 2013). En todos estos procesos de prevención desde el grupo de iguales es esencial la implicación activa por parte de los y las estudiantes, teniendo en cuenta sus motivaciones, necesidades e intereses (Redondo-Sama et al., 2014).

\section{Acciones Organizativas para la Prevención e Intervención ante el Acoso Escolar por Cuestiones de Género.}

En este apartado se plantean algunas acciones educativas desde el ámbito de la organización que hemos hallado en la revisión documental realizada y que se centran principalmente en sensibilizar, formar e implicar a toda la comunidad educativa. A continuación, exponemos algunas de ellas, partiendo siempre del primer paso, que es el de reconocer la existencia de situaciones de violencia escolar desde edades muy tempranas y de no trivializarlas (Mayes \& Cohen, 2003).

\section{Sensibilización y Formación}

Un elemento en el que coinciden todas las investigaciones internacionales es en el hecho de que hay que reconocer la existencia de violencia escolar. Es necesario no trivializar las situaciones de abuso e intimidación que se dan en la primera infancia, ya que muchas de estas situaciones derivan en actitudes violentas que posteriormente se permiten al no ser identificadas como tales (Kärnä, Voeten, Poskiparta, \& Salmivalli, 2010; Oliver, 2014; Padrós, 2014). Mayes y Cohen (2003) hacen hincapié en la necesidad de 
desmitificar la creencia de que en la primera infancia las niñas y los niños no perciben la violencia. Otros autores (Duque, 2015; Oliver, 2014; Oliver \& Valls, 2004; Smith, White \& Holland, 2003) destacan concretamente cómo las primeras relaciones que se establecen son las que más socializan y tienen mayor influencia en las relaciones futuras. En este sentido, es esencial reconocer el origen y las causas de la violencia escolar, así como las consecuencias sociales y personales que tiene en las y los estudiantes (Hong \& Espelage, 2012; Nansel et al., 2001; Olweus, 1993; Vaillancourt, 2008). Para poder llevar a cabo esta sensibilización y este acceso al conocimiento sobre la violencia escolar y de género, la formación del profesorado es clave (De Botton, Puigdellívol \& de Vicente, 2012; Teixido \& Capell, 2006), ya que la mejora educativa está relacionada con la calidad de la formación docente (Darling-Hammond, 2000; Darling-Hammond \& Bransford, 2005). Por otra parte, es necesario que esta sensibilización y formación también se lleve a cabo con las familias (Díez, Gatt \& Racionero, 2011; Driessen, Smith \& Sleegers, 2005; Epstein, 2001; Flecha, 2015) y con el alumnado (Meraviglia et al., 2003). En ese sentido, la sensibilización y formación sobre la violencia escolar ha de realizarse a toda la comunidad y tiene que abordar específicamente el ámbito de la diversidad sexual y de género, una temática que según Leonardi y Staley (2015) es abordada de forma muy escasa. Según Slesaransky-Poe (2015) para crear un ambiente seguro y que acoja a los estudiantes LGBTQ es necesaria una formación para el profesorado, la dirección, la inspección, los y las familiares, los tutores, las personas voluntarias, etc. En definitiva, cualquier persona que interaccione con estos estudiantes y sus familias. Es importante que esta formación esté basada en evidencias científicas (De Botton et al., 2012) con el fin de asegurar la calidad y la efectividad de la misma. En este sentido, la investigación científica sobre acoso escolar vinculado a violencia de género, violencia homofóbica, bifóbica y transfóbica debe ser incluida en esta formación.

\section{Diálogo y Participación de la Comunidad: Comisiones, Normas, etc.}

Uno de los elementos que plantean Teixido y Castillo (2010) es la necesidad de democratizar la vida de los centros educativos. Esto implica 
incorporar hábitos de funcionamiento organizativo que fomenten el diálogo entre profesorado, alumnado, dirección y familias con el fin de buscar la resolución pacífica de conflictos (Arribas \& Torrego, 2006). De hecho diversas investigaciones internacionales plantean la necesidad de promover la participación de toda la comunidad (American Educational Research Association, 2013; Department of Education and Training, 2015) y de establecer diálogos. Una de las acciones concretas es la creación de comisiones de convivencia (Arribas \& Torrego, 2006), en las que es importante que participen todos los estamentos de la comunidad educativa para la resolución de conflictos (Aguado \& de Vicente, 2006). El trabajo de Oliver et al. (2009) y la investigación de Valls (2005) identifican el modelo comunitario o dialógico de prevención de conflictos como una vía esencial para la prevención. En él, a través de la participación conjunta de toda la comunidad, se elaboran normas consensuadas relacionadas con la prevención de la violencia de género y toda la comunidad vela por su aplicación. Este modelo dialógico de prevención de conflictos se desarrolla en diversos centros educativos (Duque, 2015; Martin \& Tellado, 2012) y uno de las acciones que de él se derivan es la creación de una comisión mixta. Es decir, una comisión en la que participan familiares, profesorado y alumnado. Ante problemas de convivencia existentes o como prevención de los mismos, esta comisión elabora la propuesta de una norma de convivencia que se va consensuando con toda la comunidad educativa través de asambleas de familiares, profesorado y alumnado. De esta manera todas las personas que conforman la comunidad educativa se ponen de acuerdo, convirtiéndose esta en veladora del cumplimiento de la norma. Este enfoque ha sido incorporado en el Ministerio de Educación Cultura y Deporte (MECD) ${ }^{8}$.

\section{Educación y Aulas Inclusivas}

La educación inclusiva es clave para la no discriminación de ningún colectivo y la consecuente prevención de la violencia de género. Numerosos estudios (Brown \& Di Tillio, 2013; Losen \& Martínez, 2013; Losen \& Skiba, 2010; Office for Civil Rights, 2010; Skiba et al. 2011) resaltan que los grupos más vulnerables (LGTBI, inmigrantes, minorías 
étnicas, personas con discapacidad, etc.) son a menudo víctimas de acoso escolar y resaltan la importancia de actuar desde una educación inclusiva. Por su parte, Fredman, Schultz y Hoffmann (2015) examinan cómo la incorporación de prácticas inclusivas contribuye a crear escuelas seguras para estudiantes LGBTQ.

Además de las prácticas inclusivas globales del centro, también en el día a día de las aulas pueden incorporarse mecanismos organizativos inclusivos. Como plantean Teixido \& Castillo (2010) la plena integración (social y escolar) de los estudiantes es un objetivo básico como estrategia de prevención de la violencia. Desde este marco proponen prácticas para promover la inclusión como el aprendizaje cooperativo en grupos heterogéneos. Un ejemplo de organización de aula inclusiva se encuentra en los grupos interactivos (Elboj \& Niemelä, 2010; Valls \& Kyriakides, 2013) en los que niñas y niños se agrupan en grupos heterogéneos. En estos grupos, coordinados por una persona adulta, se fomentan las interacciones entre las niñas y niños, generando así no solo más aprendizajes sino también un aumento de la solidaridad; llevando a cabo una educación inclusiva.

\section{Conclusiones}

En este artículo hemos partido de la evidencia, y hemos aportado algunos de los datos existentes acerca de la violencia en los centros educativos y su vinculación con el género. La violencia de género, homofobia, transfobia y bifobia forman parte de la vida de los centros educativos igual que forman parte de la sociedad. Aun así, existen algunas barreras, como las dificultades para obtener datos sobre violencia de género, principalmente en lo relativo a los y las menores, o para estudiar las discriminaciones que sufre el colectivo LGTB, así como la invisibilización de las cuestiones de género en el concepto genérico bullying, lo que hace que los datos disponibles sean realmente escasos. Esta temática no sólo requiere ser más estudiada, sino que además visibilizar y no relativizar la existencia de la violencia escolar vinculada al género es clave para poder actuar educativamente frente a dicha violencia. 
De hecho, el reconocimiento, la sensibilización y la formación son algunos de los primeros elementos que se destacan como preventivos en la revisión de la literatura científica, resaltando la necesidad de promover una formación que incluya temas específicos de violencia de género y de LGTBfobia. También, tal como se menciona en el artículo, se hace necesario subrayar la necesidad de que dicha formación esté basada en evidencias científicas. Una formación de calidad que recoja los avances científicos en el estudio del bullying y presente aquellas actuaciones educativas que están siendo de éxito, que realmente están funcionando.

Desde el ámbito de la organización escolar, se destaca de forma continua como, ante la creciente violencia escolar, las acciones preventivas no deben quedarse en acciones puntuales con personas concretas. Es necesaria una orientación de escuela inclusiva y una democratización de los centros educativos a través de la participación de toda la comunidad. Esto pasa por promover una participación en aspectos relevantes de la vida del centro, como por ejemplo la participación en la toma de decisiones no sólo vinculada, aunque también, a los organismos establecidos para tales fines. En este sentido, cabe resaltar experiencias como el modelo dialógico de resolución de conflictos y la creación, dentro de este marco, de comisiones mixtas de convivencia (formadas por familias, profesorado, alumnado) que funcionan a través del diálogo igualitario y que se llevan a cabo en diversos centros educativos ${ }^{9}$. Este tipo de participación de toda la comunidad es la que da lugar a un liderazgo compartido que promueve acciones globales de centro.

Finalmente, se destaca la importancia de la bystander intervention, la intervención del grupo de iguales frente al bullying. Es esencial que el grupo de iguales, de la misma manera que el resto de agentes de la comunidad, rompa el silencio, denunciando las situaciones de violencia, y que se posicione claramente siempre a favor de las víctimas. Para que esto suceda, sin embargo, resulta imprescindible crear un clima de solidaridad dentro del centro educativo, que fomente la amistad entre los y las estudiantes.

En definitiva, es necesario crear un clima de centro donde haya un posicionamiento claro de toda la comunidad siempre a favor de las víctimas, y nunca a favor de quienes ejercen la violencia. Sólo de esta 
manera se podrá conseguir que ninguna persona sufra ningún tipo de acoso escolar independientemente de su género y su opción sexual.

\section{Notas}

1. En este artículo encontraremos diferentes siglas para identificar a los colectivos de Gays, Lesbianas, Bisexuales, Transgénero y Transexuales, debido a que hemos respetado como eran nombrados en los diferentes documentos consultados. Por este motivo podemos encontrar los siguientes términos: LGBT, LGTBI, LGB, LGTBQ, LGTBifobia.

2. http://www.unfe.org/es

3. http://chrysallis.org.es

4. http://www.mecd.gob.es/educacion-mecd/mc/convivencia-escolar/experiencias-deexito/otras-entidades/acoso-escolar.html

5. https://www.boe.es/boe/dias/2013/12/10/pdfs/BOE-A-2013-12886.pdf

6. https://www.boe.es/buscar/pdf/2004/BOE-A-2004-21760-consolidado.pdf

7. https://www.boe.es/boe/dias/2014/11/20/pdfs/BOE-A-2014-11990.pdf

8. http://www.mecd.gob.es/educacion-mecd/mc/convivenciaescolar/formacion/materiales.html

9. A modo de ejemplo: http://www.santiagoapostolcabanyal.es/alumnado/modelo-dialogicode-prevencion-y-resolucion-de-conflictos/

\section{Referencias}

Álvarez, R. J. (2015, 30 de diciembre). Acosaron a Alan desde los 14 años, su suicidio es un crimen social. El Mundo. Recuperado de http://www.elmundo.es/sociedad/2015/12/30/5682ca5322601d8c0f8 b4632.html

American Educational Research Association. (2013). Prevention of bullying in schools, colleges, and universities: Research report and recommendations. Washington, DC: American Educational Research Association.

Aguado, C. \& de Vicente, J. (2006). Gestión democrática de las normas. En J.C. Torrego (Coord.) Modelo integrado de mejora de la convivencia: Estrategias de Mediación y tratamiento de conflictos. Barcelona: Graó. 
194 Duque \& Teixido - Bullying, Género y Organización Escolar

Amnistía Internacional. (2008). Escuelas seguras. El derecho de cada niña.

Madrid: EDAI. Editorial Amnistía Internacional. Recuperado

https://www.es.amnesty.org/uploads/media/Spanish-

_Escuelas_Seguras-_El_Derecho_De_Cada_Nina.pdf

Arribas, J. M. \& Torrego, J.C. (2006). El modelo integrado. Fundamentos, estructuras y su despliegue en la vida de los centros. En J. C. Torrego, (coord.), Modelo integrado de mejora de la convivencia. Estrategias de mediación y tratamiento de conflictos. Barcelona: Editorial Graó.

Banks, R. (1997). Bullying in schools (ERIC Digest ED407154).

Champaign, IL: ERIC Clearinghouse on Elementary and Early Childhood Education.

Banyard, V. L., Plante, E. G., Cohn, E. S., Moorhead, C., Ward, S., \& Walsh, W. (2005). Revisiting Unwanted Sexual Experiences on Campus A 12-Year Follow-Up. Violence Against Women, 11(4), 426-446. doi:10.1177/1077801204274388

Boulton, M. J. (1999). Concurrent and longitudinal relations between children's playground behavior and social preference, victimization, and bullying. Child Development, 7(4), 944-954. doi:10.1111/14678624.00068

Brown, C. A., \& Di Tillio, C. (2013). Discipline disproportionality among Hispanic and American Indian students: Expanding the discourse in US research. Journal of Education and Learning, 2(4), 47-59. doi:10.14507/epaa.v8n1.2000

Cava, M., Buelga, S., Musitu, G., \& Murgui, S. (2010). Violencia escolar entre adolescentes y sus implicaciones en el ajuste psicosocial: un estudio longitudinal. Revista de Psicodidáctica, 15(1), 21-34.

Recuperado de: http://www.redalyc.org/articulo.oa?id=17512968002 Darling-Hammond, L. (2000). Teacher Quality and Student Achievement: A Review of State Policy Evidence. Education Policy Analysis Archives, 8, 1. doi:10.14507/epaa.v8n1.2000

Darling-Hammond, L., \& Bransford, J. (Ed). (2005). Preparing Teachers for a Changing World: What Teachers Should Learn and Be Able to Do. National Academy of Education, Committee on Teacher Education. San Francisco: Jossey Bass. 
De Botton, L., Puigdellívol, I., \& de Vicente, I. (2012). Evidencias científicas para la formación inicial del profesorado en prevención y detección precoz de violencia de género. Revista Interuniversitaria de Formación del Profesorado, 73(26,1), 41-55. Recuperado de http://www.aufop.com/aufop/uploaded_files/articulos/1396510014.p df

Delegación del Gobierno para la Violencia de Género. (2015). Portal Estadístico para la Violencia de género. Recuperado de http://estadisticasviolenciagenero.msssi.gob.es/

Department of Education and Training. (2015). A review of literature (2010-2014) on student bullying by Australia's Safe and Supportive School Communities Working Group. Queensland: Department of Education and Training. Recuperado de http://news.bullyingnoway.gov.au/thefacts/Snapshots/Literature\%20Review\%20Bullying\%20by\%20SSSC. pdf

Devine, J., \& Cohen, J. (2007) Making your school safe: Strategies to protect children and promote learning. Nueva York: Teachers College Press.

Díaz-Aguado, M. J. (Dir.). (2004). Prevención de la violencia y lucha contra la exclusión desde la adolescencia. Madrid: Instituto de la Juventud.

Díaz-Aguado, M. J. (2006). Sexismo, violencia de género y acoso escolar. Propuestas para una prevención integral de la violencia. Revista de Estudios de Juventud, 6(73), 38-58. Recuperado de http://www.injuve.es/sites/default/files/revista73_3.pdf

Díaz-Aguado, M. J., \& Martínez, R. (2001). La construcción de la igualdad y la prevención de la violencia contra la mujer desde la educación secundaria. Madrid: Instituto de la Mujer.

Díaz-Aguado, M. J., \& Carvajal, M. I. (2011). Igualdad y prevención de la violencia de género en la adolescencia. Madrid: Ministerio de Igualdad.

Díez, J., Gatt, S., \& Racionero, S. (2011). Placing immigrant and minority family and community members at the school's centre: The role of 
196 Duque \& Teixido - Bullying, Género y Organización Escolar

community participation. European Journal of Education, 46(2), 209-218. doi:10.1111/j.1465-3435.2011.01474.x

Driessen, G., Smith, F., \& Sleegers, P. (2005). Parental involvement and educational achievement. British Educational Research Journal, 31(4), 509-532. doi:10.1080/10852352.2010.486297

Duque, E. (Coord.) (2015). IDEALOVE\&NAM. Socialización preventiva de la Violencia de Género. Madrid: CNIIE.

Edwards, A., \& Warin, J. (1999). Parental involvement in raising the achievement of primary school pupils: Why bother? Oxford Review of Education, 25(3), 325-341. doi:10.1080/030549899104017

Elboj, C., \& Niemelä, R. (2010). Sub-Communities of Mutual Learners in the Classroom: The case of Interactive Groups. Revista de Psicodidáctica, 15(2), 177-189. doi:10.1387/RevPsicodidact.810

Epstein, J. L. (2001). School, Family and Community Partnerships:

Preparing educators and improving schools. Boulder: Westview Press.

Espelage, D. L., Astor, R. A., Cornell, D., Lester, J., Mayer, M. J., \& Meyer, E. J. (2013). Prevention of bullying in schools, colleges, and universities. Washington, DC: American Educational Research Association.

European Union Agency for Fundamental Rights-FRA. (2014). Victims of crime in EU: The extent and nature of support for victims.

Recuperado de http://fra.europa.eu/en/publication/2014/victimscrime-eu-extent-and-nature-support-victims

Federación Estatal de Lesbianas, Gais, Transexuales y Bisexuales. (2012) Acoso escolar homofóbico y riesgo de suicidio en adolescentes y jóvenes LGB. FELGTB: Madrid. Recuperado de:

http://www.felgtb.org/rs/1584/d112d6ad-54ec-438b-93584483f9e98868/91c/filename

Fernández, D. (2008, 6 de noviembre). La menor de Ripollet murió por "colgar" un video besándose con su presunto agresor. 20 minutos. Recuperado de http://www.20minutos.es/noticia/426224/0/ripollet/maore/asesinato/ 
Fineran, S., \& Bennett, L. (1999). Gender and power issues of peer sexual harassment among teenagers. Journal of Interpersonal Violence, 14(6), 626-641. doi:10.1177/088626099014006004

Fisher, B. S., Daigle, L. E., \& Cullen, F. T. (2010). What Distinguishes Single from Recurrent Sexual Victims? The Role of Lifestyle-Routine Activities and First-Incident Characteristics. Justice Quarterly, 27(1), 102-129. doi:10.1080/07418820902763061 Flecha, R. (2015). Successful Educational Actions for Inclusion and Social Cohesion in Europe. New York: Springer.

Fredman, A., Schultz, N., \& Hoffman, M. F. (2015) "You're Moving a Frickin' Big Ship": The Challenges of Addressing LGBTQ Topics in Public Schools. Education and Urban Society, 47(1) 56-85. doi:10.1177/0013124513496457

Fundación ANAR. (2015). Informe Violencia de Género 2014. Recuperado de http://www.anar.org/wp-content/uploads/2015/05/InformeTel\%C3\%A9fono-ANAR-Violencia-G\%C3\%A9nero-2014.pdf

FUNDADEPS. Fundación de Educación para la Salud. (2012). Cómo construir una escuela saludable y segura. Guía para padres y madres. Madrid: CEAPA. Confederación Española de Asociaciones de Padres y Madres de Alumnos.

Gairín, J., Armengol, C., \& Silva, B. P. (2013) El "Bullying” escolar. Consideraciones organizativas y estrategias para la intervención. Educación XX1, 16(1) 17-38. doi:10.5944/educXX1.16.1.715

Gómez, J. (2004). El amor en la sociedad del riesgo. Una tentativa educativa. Barcelona: El Roure.

Gómez, A., Munté, A., \& Sordé, T. (2014). Transforming schools through minority males' participation: Overcoming cultural stereotypes and preventing violence. Journal of Interpersonal violence, 29(11), 20022020. doi: $10.1177 / 0886260513515949$

Gregory, A., \& Cornell, D. (2009). "Tolerating” adolescent needs: Moving beyond zero tolerance policies in high school. Theory into Practice, 48(2), 106-113. doi:10.1080/00405840902776327

Gross, A. M., Winslett, A., Roberts, M., \& Gohm, C. L. (2006). An examination of sexual violence against college women. Violence against Women, 12(3), 288-300. doi:10.1177/1077801205277358 
198 Duque \& Teixido - Bullying, Género y Organización Escolar

Guo, P., \& Higgins-D'Alessandro, A. (2011). The place of teachers' views of teaching in promoting positive school culture and student prosocial and academic outcomes. Paper presented at the Association for Moral Education annual conference, Nanjing, China 24-28 October 2011.

Hong, J. S., \& Espelage, D. L. (2012). A review of research on bullying and peer victimization in school: An ecological system analysis. Aggression and Violent Behavior, 17(4), 311-322 doi:10.1016/j.avb.2012.03.003

Houston, P. F., Blankstein, A. M., \& Cole, R.W. (2010). Leadership for Family and Community Involvement (The Soul of Educational Leadership Series). US: HOPE Foundation and the American Association of School Administrators.

Instituto Nacional de Estadística. (2015). Estadística de Violencia Doméstica y Violencia de Género Año 2014. Recuperado de http://www.ine.es/prensa/np906.pdf

Jia, Y., Way, N., Ling, G., Yoshikawa, H., Chen, X., Hughes, D., \& Lu, Z. (2009). The influence of student perceptions of school climate on socio-emotional and academic adjustment: A comparison of Chinese and American adolescents. Child Development, 80(5), 1514-1530. doi:10.1111/j.1467-8624.2009.01348.x.

Kärnä, A., Voeten, M., Poskiparta, E., \& Salmivalli, C. (2010). Vulnerable children in varying classroom contexts: Bystanders' behaviors moderate the effects of risk factors on victimization. Merrill-Palmer Quarterly, 56(3), 261-282 Recuperado de http://www.jstor.org/stable/23098070

Lavoie, F., Robitaille, L., \& Hébert, M. (2000). Teen dating relationships and aggression. An exploratory study. Violence against Women, 6(1), 6-36. doi:10.1177/10778010022181688

Leithwood, K., Day, C., Sammons, P., Harris, A., \& Hopkins, D. (2006). Seven strong claims about successful school leadership. Nottingham: National College of School Leadership.

Leonardi, B., \& Staley, S. (2015). Affirm gender and sexual diversity within the school community. Phi Delta Kappan, 97(3), 69-73. doi:10.1177/0031721715614832 
Losen, D. J., \& Martinez, T. E. (2013). Out of School and Off Track: The Overuse of Suspensions in American Middle and High Schools. UCLA: Civil Rights Project.

Losen, D. J., \& Skiba, R. J. (2010). Suspended education: Urban middle schools in crisis. UCLA: Civil Rights Project

Mayberry, M., Chenneville, T., \& Currie, S. (2013) Challenging the Sounds of Silence: A Qualitative Study of Gay-Straight Alliances and School Reform Efforts. Education and Urban Society, 45(3), 307339. doi: $10.1177 / 0013124511409400$

Mayes, L. C., \& Cohen, D. J. (2003). Guía para entender a tu hijo del Centro Yale de Estudios Infantiles: Un desarrollo sano desde el nacimiento a la adolescencia. Madrid: Alianza Editorial.

Martín, N. \& Tellado, I. (2012). Violencia de Género y Resolución Comunitaria de Conflictos en los Centros Educativos. GÉNEROS. Multidisciplinary Journal of Gender Studies, 1(3), 300-319. doi:10.4471/generos.2012.14

McGuire, J. K., Anderson, C. R., Toomey, R. B., \& Russell, S. T. (2010). School climate for transgender youth. A mixed method investigation of student experiences and school responses. Journal of Youth Adolescence, 39, 1175-1188. doi:10.1007/s10964-010-9540-7 Meraviglia, M. G., Becker, H., Rosenbluth, B., Sanchez, E., \& Robertson, T. (2003). The Expect Respect Project: Creating a positive elementary school climate. Journal of Interpersonal Violence, 18(11), 1347-60. doi:10.1177/0886260503257457

Monge, Y. (2012, 17 de octubre). Un caso de ciberacoso conmociona a la sociedad canadiense. El País. Recuperado de

http://sociedad.elpais.com/sociedad/2012/10/17/actualidad/13505066 05_509352.html

Nansel, T. R., Overpeck, M., Pilla, R. S., Ruan, W. J., Simons-Morton, B., \& Scheidt, P. (2001). Bullying behaviors among US youth: Prevalence and association with psychosocial adjustment. Jama, 285(16), 2094-2100. Recuperado de http://www.ncbi.nlm.nih.gov/pmc/articles/PMC2435211/ Observatori contra l'Homofòbia. (2015). L'estat de la LGTBIfòbia a Catalunya 2015. Barcelona: Observatori contra l'homofòbia. 
200 Duque \& Teixido - Bullying, Género y Organización Escolar

Recuperado de http://observatori-contra-

homofobia.blogspot.com.es/2016/04/estudi-oficial-och-lestat-dela.html

Office for Civil Rights, U.S. Department of Education. (2010). "Dear

Colleague" letter. Washington, DC: Office for Civil Rights, U.S.

Department of Education. Recuperado de

http://www2.ed.gov/about/offices/list/ocr/letters/colleague-

201010.html

Oliver, E., Soler, M., \& Flecha, R. (2009). Opening schools to all (women): efforts to overcome gender violence in Spain. British Journal of Sociology of Education, 30(2), 207-218.

doi:10.1080/01425690802700313

Oliver, E. (2014). Zero Violence Since Early Childhood The Dialogic Recreation of Knowledge. Qualitative Inquiry, 20(7), 902-908 doi:10.1177/1077800414537215

Oliver, E., \& Valls, R. (2004). Violencia de Género: investigaciones sobre quiénes, por qué y cómo superarla. Barcelona: El Roure.

Olweus, D. (1993). Acoso escolar, "bullying", en las escuelas: hechos e intervenciones. Bergen: Centro de investigación para la Promoción de la Salud, Universidad de Bergen.

O'Moore, A. M., \& Minton, S. J. (2005). Evaluation of the effectiveness of an anti-bullying programme in primary schools. Aggressive Behavior 31, 609-622. doi:10.1002/ab.20098

Organización Mundial de la Salud. (2013). Informe de la OMS destaca que la violencia contra la mujer es "un problema de salud global de proporciones epidémicas". Recuperado de

http://www.who.int/mediacentre/news/releases/2013/violence_against_wo men_20130620/es/

Osvaldsson, K. (2011). Bullying in context: Stories of bullying on an Internet discussion board. Children \& Society, 25(4), 317-327. doi:10.1111/j.1099-0860.2011.00383.x

Padrós, M. (2014). A Transformative Approach to Prevent Peer Violence in Schools Contributions from Communicative Research Methods. Qualitative Inquiry, 20(7), 916-922. doi:10.1177/1077800414537217 
Padrós, M., \& Flecha, R. (2014). Towards a Conceptualization of Dialogic Leadership. International Journal of Educational Leadership and Management, 2(2), 207-226. doi:10.4471/ijelm.2014.17

Piñuel, I., \& Oñate, A. (2007). Acoso y Violencia Escolar en España: Informe Cisneros $X$. Madrid: IIEDDI.

Piulachs, M. (2015, 7 de diciembre). El registre civil canvia el nom a dos menors transsexuals Catalans. El Punt Avui. Recuperado de http://www.elpuntavui.cat/societat/article/5-societat/921346-elregistre-civil-canvia-el-nom-a-dos-menors-transsexuals-catalans.html

Redondo-Sama, G., Pulido-Rodríguez, M. A., Larena, R., \& De Botton, L. (2014). Not Without Them. The Inclusion of Minors' Voices on Cyber Harassment Prevention. Qualitative Inquiry, 20(7) 895-901. doi:10.1177/1077800414537214

Redondo-Sama, G. (2015). Liderazgo dialógico en comunidades de aprendizaje. Intangible Capital, 11(3), 437-457. doi:10.3926/ic.651

Robinson, V., Lloyd, C. A., \& Rowe, K. J. (2008). The Impact of Leadership on Student Outcomes: An Analysis of the Differential Effects of Leadership Types. Educational Administration Quarterly, 44(5), 635-674. doi:10.1177/0013161X08321509.

Sanders, M. G., \& Harvey, A. (2002). Beyond the school walls: A case study of principal leadership for school-community collaboration. Teachers College Record, 104(7), 1345-1368. doi:10.1111/14679620.00206

Sanders, M. G., \& Sheldon, S. B. (Eds.) (2009). Principals matter: A guide to school, family, and community partnerships. Thousand Oaks, CA: Corwin Press.

Sénéchal, M., \& LeFevre, J. A. (2002). Parental involvement in the development of children's reading skill: A five-year longitudinal study. Child Development, 73(2), 445-460. doi:10.1111/14678624.00417

Silverman, J.G., Raj, A., Mucci, L. A., \& Hathaway, J. E. (2001). Dating violence against girls and associated substance use, unhealthy weight control, sexual risk behavior, pregnancy and suicidality. JAMA, 286(5), 572-579. doi:10.1001/jama.286.5.572. 
Skiba, R. J., Horner, R. H., Chung, C. G., Karega Rausch, M., May, S. L., \& Tobin, T. (2011). Race is not neutral: A national investigation of African American and Latino disproportionality in school discipline. School Psychology Review, 40(1), 85-107. Recuperado de http://www.indiana.edu/ atlantic/wp-content/uploads/2011/12/Skibaet-al.-Race-is-not-neutral..pdf

Slesaransky-Poe, G. (2013). Adults Set the Tone for welcoming all Students. Phi Delta Kappan, 94(5), 40-44. doi:10.1177/003172171309400509

Smith, P. H., White, J. W., \& Holland, L. J. (2003). A longitudinal perspective on dating violence among adolescent and college-age women. American Journal of Public Health, 93(7), 1104-1109. doi:10.2105/AJPH.93.7.1104

Srabstein, J. C. (2015). The global implications of bullying and other forms of maltreatment, in the context of migratory trends and psychiatric resources. Child and adolescent psychiatric clinics of North America, 24(4), 799-810. doi:10.1016/j.chc.2015.06.008

Teixido, J., \& Capell, D. (2006). La millora de la convivència als centres educatius. Què poden fer-hi els equips directius. I Jornada d'organització i Direcció de Centres Educatius. Girona 14 de novembre de 2006. Recuperado de http://www.joanteixido.org/doc/convivencia/poden_fer_directius.pdf

Teixido, J., \& Castillo, M. (2010). Prácticas de mejora de la convivencia escolar. Recopilación y análisis. Málaga: Aljibe.

Thapa, A., Cohen, J., Guffey, S., \& Higgins-D'Alessandro, A. (2013). A review of school climate research. Review of Educational Research, 83(3), 357-385. doi:10.3102/0034654313483907

Torrego, J. C., \& Martínez, C. (2014). Claves para el Desarrrollo del Plan de Convivencia en los Centros Educativos desde una Perspectiva Integral. Qualitative Research in Education, 3(1), 83-113. doi:10.4471/qre.2014.37

UNFE. (n.f). Ficha de datos. Violencia homofóbica y transfóbica. UNFE. Recuperado de https://www.unfe.org/system/unfe-22UN_Fact_Sheets_-_Spanish_v1d.pdf 
UNICEF. (2014). Hidden in plain sight: A statistical analysis of violence against children. Recuperado de http://files.unicef.org/publications/files/Hidden_in_plain_sight_statist ical_analysis_EN_3_Sept_2014.pdf

Vaillancourt, T., McDougall, P., Hymel, S., Krygsman, A., Miller, J., Stiver, K., \& Davis, C. (2008). Bullying: Are researchers and children/youth talking about the same thing? International Journal of Behavioral Development, 32(6), 486-495.

doi:10.1177/0165025408095553

Valls, R. (2005). Prevenció de la violència de gènere en el context educatiu a partir de noves formes d'organització escolar [Prevención de la violencia de género en el contexto educativo a partir de nuevas formes de organización escolar]. Barcelona: Institut Català de les Dones.

Valls, R., \& Kyriakides, L. (2013). The power of Interactive Groups: how diversity of adults volunteering in classroom groups can promote inclusion and success for children of vulnerable minority ethnic populations. Cambridge Journal of Education, 43(1), 17-33. doi:10.1080/0305764X.2012.749213

Wagner, A., \& Magnusson, J. L. (2005). Neglected realities: exploring the impact of women's experiences of violence on learning in sites of higher education. Gender and Education, 17(4), 449-461. doi:10.1080/09540250500145189

Wang, M. T., Selman, R. L., Dishion, T. J., \& Stormshak, E. A. (2010). A Tobit regression analysis of the covariation between middle school students' perceived school. Journal of Research on Adolescence, 20(2), 274-286. doi:10.1111/j.1532-7795.2010.00648.x

Whittle, S., Turner, L., Combs, R., \& Rhodes, S. (2008). Transgender EuroStudy. Legal Survey and Focus on the Transgender Experience of Health Care. Brussels: ILGA EUROPE. Recuperado de http://tgeu.org/wp-content/uploads/2009/11/transgender_web.pdf

Witziers, B., Bosker, R.J., \& Kruger, M.L. (2003). Educational leadership and student achievement: The elusive search for an association. Educational Administration Quarterly, 39(3), 398-425. doi:10.1177/0013161X03253411 
204 Duque \& Teixido - Bullying, Género y Organización Escolar

Yoneyama, S., \& Naito, A. (2003). Problems with the paradigms: The school as a factor in understanding bullying (with special reference to Japan). British Journal of Sociology of Education, 24(3), 315-30. doi:10.1080/01425690301894

Elena Duque es profesora del Departamento de Pedagogía de la Universidad de Girona.

Joan Teixido es profesor titular del Departamento de Pedagogía de la Universidad de Girona.

Contact Address: Facultad de Educación y Psicología, Universidad de Girona. Plaça Sant Domènec 9, 17004, Girona

E-mail: elena.duque@udg.edu 